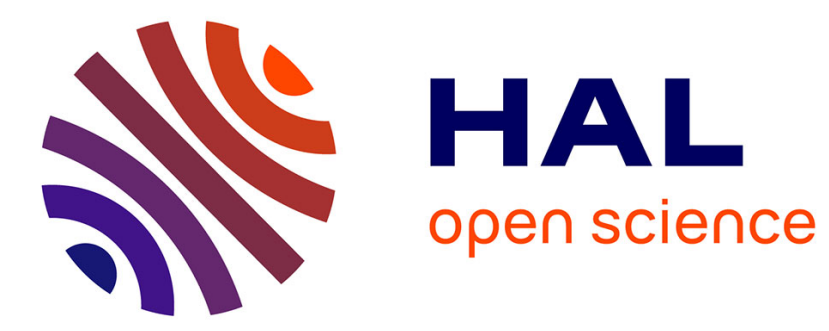

\title{
Robust output feedback MPC for LPV systems using interval observers
}

\author{
Alex dos Reis de Souza, Denis Efimov, Tarek Raïssi
}

\section{To cite this version:}

Alex dos Reis de Souza, Denis Efimov, Tarek Raïssi. Robust output feedback MPC for LPV systems using interval observers. IEEE Transactions on Automatic Control, 2021, 10.1109/TAC.2021.3099449 . hal-03220490

\section{HAL Id: hal-03220490 \\ https://hal.inria.fr/hal-03220490}

Submitted on 7 May 2021

HAL is a multi-disciplinary open access archive for the deposit and dissemination of scientific research documents, whether they are published or not. The documents may come from teaching and research institutions in France or abroad, or from public or private research centers.
L'archive ouverte pluridisciplinaire HAL, est destinée au dépôt et à la diffusion de documents scientifiques de niveau recherche, publiés ou non, émanant des établissements d'enseignement et de recherche français ou étrangers, des laboratoires publics ou privés. 


\title{
Robust output feedback MPC for LPV systems using interval observers
}

\author{
Alex Reis de Souza, Denis Efimov, and Tarek Raïssi
}

\begin{abstract}
This work addresses the problem of robust output feedback model predictive control for discrete-time, constrained, linear parameter-varying systems subject to (bounded) state and measurement disturbances. The vector of scheduling parameters is assumed to be an unmeasurable signal taking values in a given compact set. The proposed controller incorporates an interval observer, that uses the available measurement to update the setmembership estimation of the states, and an interval predictor, used in the prediction step of the MPC algorithm. The resulting MPC scheme offers guarantees on recursive feasibility, constraint satisfaction, and input-to-state stability in the terminal set. Furthermore, this novel algorithm shows low computation complexity and ease of implementation (similar to conventional MPC schemes).
\end{abstract}

Index Terms-Predictive control, robust control, output feedback

\section{INTRODUCTION}

The problem of control and observation of nonlinear systems has been extensively studied over the last decades, becoming an important branch of research on automatic control [1]. Among the numerous existing techniques to solve such problems, the ones involving linear parameter-varying (LPV) models have received special attention [2]. An LPV representation of a nonlinear system consists in a family of linear time-invariant (LTI) systems, which are interpolated by a scheduling parameter that can indicate, for instance, some operating points of the nonlinear dynamics. In this framework, design methods developed for LTI systems can be applied.

However, even considering a linear-like system, the problem of controlling dynamical plants under constraints is very difficult - or even impossible - to be tackled using classical state feedback tools [3]. This is one of the reasons why Model Predictive Control (MPC) became popular in the last decades: by solving an (online) optimal control problem, it handles constraints on states, inputs and outputs. This control problem consists of minimizing an optimal criterion that incorporates a finite window of predictions of the future behaviour of the system, computed by a (possibly multivariate) discrete-time model [4].

Robustness has also been an important topic when dealing with MPC [5]. In practice, the plants are often plagued by uncertainties (such as bounded disturbances, noise and parametric variations), causing discrepancies between the model and the real system. These errors might lead the behaviour of the latter to be very different from the predictions, possibly causing deterioration of performance, constraint transgression or even instability. In this sense, an MPC scheme is said to be robust if it achieves the control task while

This work was partially supported by the IPL COSY, and by the Ministry of Science and Higher Education of Russian Federation, passport of goszadanie no. 2019-0898.

Alex Reis de Souza is with Inria - Lille Nord Europe, Université de Lille CNRS, UMR 9189 - CRIStAL, F-59000 Lille, France. (e-mail: alex.dosreis-de-souza@inria.fr)

Denis Efimov is with Inria - Lille Nord Europe, Université de Lille CNRS, UMR 9189 - CRIStAL, F-59000 Lille, France, and with ITMO University, 49 av. Kronverkskiy, 197101 Saint Petersburg, Russia. (email: denis.efimov@inria.fr)

Tarek Raïssi is with Conservatoire National des Arts et Métiers (CNAM), Cedric 292, Rue St-Martin, 75141 Paris, France. (e-mail: tarek.raissi@cnam.fr) satisfying the constraints for all possible realizations of perturbations in a certain range [6].

Furthermore, since MPC requires full-state measurement (which is not always available), the case where only output feedback is available makes it necessary to rely on state estimation tools [6]. The inherent estimation error adds even more uncertainty to the problem [5]. For linear systems, the literature on robust output feedback MPC is mature, and the LPV case has been an active field of research over the last decade. The main difficulty in this scenario comes from the fact that, even if the scheduling parameter is measured online, it is hard to predict its evolution in future steps - and consequently, to obtain a reliable prediction of the real system [7].

Nevertheless, several dynamic output feedback controllers have been proposed over the last years, e.g., Ding et al. [8] [9]. In a different approach, [10] proposes an observer-based technique relying on input-to-state stability and robust positively invariant sets of the estimation error. In [11], the authors develop an approach that optimizes, simultaneously, both controller and observer. A tubebased method is presented by [12]. Min-max optimization has been utilized in [13] and [14], although no state constraints are imposed. Most of these works recursively update the estimation error sets and thus require the common assumption that the scheduling parameter is measured, (there are exceptions, e.g., [15], [16], but with a constrained prediction horizon).

In the present paper, following an idea recently proposed for LTI systems [17], we present an alternative for robust output feedback MPC for LPV systems using interval observers (IO) [18]. These observers - while being a special class of set-membership estimators - provide a guaranteed estimation of the admissible values (i.e., intervals) of the states, by using input-output information at each instant of time. The design of IOs, while based on the concept of positive systems, is a mature topic and covers many kinds of systems and applications (see, for instance, survey [19]).

This paper proposes an output feedback MPC scheme for LPV systems, without measurement of the scheduling parameters, with guarantees on stability and constraint satisfaction. By incorporating an interval predictor (IP), the MPC evaluates an interval in which the system's trajectories evolve, using only information on the disturbance bounds and the set of admissible values for the scheduling parameters. The gains of the IO and IP, as well as for the terminal controller (which is designed concerning the interval predictor), are obtained by solving (offline) LMIs, while the terminal set is readily evaluated as an ellipsoid. Finally, the optimization problem to be solved online is convex.

The paper is organized as follows: Section II gives the problem statement, the design and the features of the interval estimators are presented in Section III, Section IV introduces the MPC algorithm and its properties on stability and guarantees on constraint satisfaction, as well as a discussion on complexity and its contrast with the existing literature. Section V illustrates the proposed approach with a numerical example. Finally, Section VI concludes this study, also giving future directions of research.

Notation:

- The sets of real and integer numbers are defined by $\mathbb{R}$ and $\mathbb{Z}$, respectively, then $|\cdot|$ represents the absolute value for an element of these sets; $\mathbb{R}_{+}=\{s \in \mathbb{R}: s \geq 0\}$ and $\mathbb{Z}_{+}=\mathbb{Z} \cap \mathbb{R}_{+}$. The 
Euclidean norm of a vector $x \in \mathbb{R}^{n}$ is denoted by $\|x\|$.

- A matrix $M$ is said to be non-negative if all of its elements are non-negative. A matrix $M$ is said to be Schur stable if all of its eigenvalues have magnitude less than one. The identity matrix of dimension $n$ is defined by $\mathbb{I}_{n}$. For a symmetric matrix $A$, the symmetric entry (i.e., $A_{i, j}=A_{j, i}$ ) is denoted by $\star$. We denote $A=\operatorname{diag}\left(a_{1}, \ldots, a_{n}\right)$ and $V=\operatorname{vec}\left(v_{1}, \ldots, v_{n}\right) \in \mathbb{R}^{\tilde{n}}$ as the diagonal matrix with block entries $A_{i i}=a_{i}$, and the vector composed by the concatenation of each vector $v_{i} \in \mathbb{R}^{n_{i}}, \tilde{n}=$ $\sum_{i=1}^{n} n_{i}$, respectively.

- For a function $x: \mathbb{Z}_{+} \rightarrow \mathbb{R}^{n}$, we use the convention $x_{k}=x(k)$ and denote $|x|_{\infty}=\sup _{k \in \mathbb{Z}_{+}}\left\|x_{k}\right\|$. Furthermore, we define $\ell_{\infty}^{n}$ as a sequence space such that, for any of its elements, we have $|x|_{\infty}<\infty$

- Let $x_{1}, x_{2} \in \mathbb{R}^{n}$ be two vectors and $A_{1}, A_{2} \in \mathbb{R}^{n \times n}$ be two matrices, then the relations $x_{1} \leq x_{2}$ and $A_{1} \leq A_{2}$ are to be understood component-wise. For a matrix $A$ (and similarly for vectors), we define $A^{+}=\max \{0, A\}, A^{-}=A^{+}-A$ (also understood component-wise), and also denote the matrix of absolute values of all elements by $|A|=A^{+}+A^{-}$. Furthermore, for a symmetric matrix $A \in \mathbb{R}^{n \times n}$ the relation $A \prec 0$ (resp. $A \succeq 0$ ) means that $A \in \mathbb{R}^{n \times n}$ is negative (resp. positive semi-) definite.

\section{Problem statement}

Consider the following discrete-time LPV system:

$$
\begin{aligned}
x_{k+1} & =A\left(\theta_{k}\right) x_{k}+B\left(\theta_{k}\right) u_{k}+w_{k} \\
y_{k} & =C x_{k}+v_{k}, \quad k \in \mathbb{Z}_{+}
\end{aligned}
$$

where $x_{k} \in \mathbb{R}^{n}$ is the state vector, $u_{k} \in \mathbb{R}^{m}$ is the control input, $y_{k} \in \mathbb{R}^{p}$ is the (measured) output, $w_{k}$ and $v_{k}$ are, respectively, state disturbance and measurement noise. The time-varying signal $\theta_{k} \in \Theta \subset \mathbb{R}^{r}$ is the scheduling parameter for the LPV system. It is assumed that $\theta_{k}$ is not measured, but its set of admissible values $\Theta$ is known. Furthermore, the matrix functions $A: \Theta \rightarrow \mathbb{R}^{n \times n}$ and $B: \Theta \rightarrow \mathbb{R}^{n \times m}$ are locally bounded and known. The measurement matrix $C \in \mathbb{R}^{p \times n}$ is assumed to be known.

Assumption 1: There exist matrices $A_{0} \in \mathbb{R}^{n \times n}, B_{0} \in \mathbb{R}^{n \times m}$ and $\Delta A_{i} \in \mathbb{R}^{n \times n}, \Delta B_{i} \in \mathbb{R}^{n \times m}, i=1, \ldots, \nu$ for some $\nu \in \mathbb{Z}_{+}$, such that the following relations are satisfied for all $\theta \in \Theta$ :

$$
\begin{gathered}
A(\theta)=A_{0}+\sum_{i=1}^{\nu} \lambda_{i}(\theta) \Delta A_{i}, \quad B(\theta)=B_{0}+\sum_{i=1}^{\nu} \lambda_{i}(\theta) \Delta B_{i}, \\
\sum_{i=1}^{\nu} \lambda_{i}(\theta)=1, \quad \lambda_{i}(\theta) \in[0,1] .
\end{gathered}
$$

Assumption 2: It is assumed that $\Delta B_{i} \geq 0$ for all $i=1, \ldots, \nu$. Assumption 1 (which is technical and it is introduced to simplify the writing) states that system (1) admits a convex embedding in a polytope defined by $\nu$ known vertices $\Delta A_{i}$ and $\Delta B_{i}$ with known centers $A_{0}, B_{0}$. Note that, since functions $A, B$ and the set $\Theta$ are known, then there exists matrices $\underline{A}, \bar{A}, \underline{B}, \bar{B}$ such that

$$
\underline{A} \leq A(\theta) \leq \bar{A}, \quad \underline{B} \leq B(\theta) \leq \bar{B}, \quad \forall \theta \in \Theta .
$$

Assumption 3: Initial conditions of (1) are bounded such as $\underline{x}_{0} \leq$ $x_{0} \leq \bar{x}_{0}$, for some known $\underline{x}_{0}, \bar{x}_{0} \in \mathbb{R}^{n}$. Furthermore, the additive perturbations $w_{k} \in\left[\underline{w}_{k}, \bar{w}_{k}\right]$ and $v_{k} \in\left[\underline{v}_{k}, \bar{v}_{k}\right]$ for all $k \in \mathbb{Z}_{+}$, where $\underline{w}, \bar{w} \in \ell_{\infty}^{n}$ and $\underline{v}, \bar{v} \in \ell_{\infty}^{p}$.

Assumption 3 is usual in the design of IOs and means that the sources of uncertainty in (1), i.e., $x_{0}, w_{k}$ and $v_{k}$, are enclosed in bounded intervals. Furthermore, we impose the following mild hypothesis concerning $C$ (which can be always achieved by a proper change of coordinates):
Assumption 4: Let $C \geq 0$.

Denote $\mathbb{X} \subset \mathbb{R}^{n}$ and $\mathbb{U} \subset \mathbb{R}^{m}$ as the convex sets of admissible values for the state and control, respectively.

Problem 1: Let $\left[\underline{x}_{0}, \bar{x}_{0}\right] \in \mathbb{X}$ and assumptions $1-3$ be satisfied. The objective is to design an output feedback controller stabilizing the LPV system (1) in a vicinity of the origin while satisfying state and control constraints, namely,

$$
x_{k} \in \mathbb{X}, \quad u_{k} \in \mathbb{U}, \quad \forall k \in \mathbb{Z}_{+}
$$

for any admissible realization of disturbances $w_{k}$ and $v_{k}$, and for all $\theta_{k} \in \Theta$.

To address this control problem, we propose an MPC algorithm that incorporates interval observers/predictors in the design. The main interest of such a choice resides on the fact that, thanks to cooperativity features and under Assumption 3, the IO/IP generates estimates $\underline{x}_{k}, \bar{x}_{k} \in \mathbb{R}^{n}$ such that the relation

$$
\underline{x}_{k} \leq x_{k} \leq \bar{x}_{k} \quad \forall k \in \mathbb{Z}_{+}
$$

is satisfied. Hence, this information can be easily used to check fulfillment of state constraints, since $\left[\underline{x}_{k}, \bar{x}_{k}\right] \subset \mathbb{X} \Rightarrow x_{k} \subset \mathbb{X}$.

\section{Preliminaries}

For our developments, we will need the following lemmas:

Lemma 1: [20] Let $x \in \mathbb{R}^{n}$ be a vector variable, $\underline{x} \leq x \leq \bar{x}$ for some $\underline{x}, \bar{x} \in \mathbb{R}^{n}$. Then,

(1) if $A \in \mathbb{R}^{m \times n}$ is a constant matrix, then

$$
A^{+} \underline{x}-A^{-} \bar{x} \leq A x \leq A^{+} \bar{x}-A^{-} \underline{x}
$$

(2) if $A \in \mathbb{R}^{m \times n}$ is a matrix variable and $\underline{A} \leq A \leq \bar{A}$ for some $\underline{A}, \bar{A} \in \mathbb{R}^{m \times n}$, then

$$
\begin{gathered}
\underline{A}^{+} \underline{x}^{+}-\bar{A}^{+} \underline{x}^{-}-\underline{A}^{-} \bar{x}^{+}+\bar{A}-\bar{x}^{-} \leq A x \\
\leq \bar{A}^{+} \bar{x}^{+}-\underline{A}^{+} \bar{x}^{-}-\bar{A} \underline{x}^{+}+\underline{A}^{-} \underline{x}^{-}
\end{gathered}
$$

Lemma 2: [21] For $A \in \mathbb{R}_{+}^{n \times n}$, the system

$$
x_{k+1}=A x_{k}+\omega_{k}, \omega: \mathbb{Z}_{+} \rightarrow \mathbb{R}_{+}^{n}, \omega \in \mathcal{L}_{\infty}^{n}, k \in \mathbb{Z}_{+}
$$

has a non-negative solution $x_{k} \in \mathbb{R}_{+}^{n}$ for all $k \in \mathbb{Z}_{+}$provided that $x_{0} \geq 0$.

Lemma 3: [22] A matrix $A \in \mathbb{R}_{+}^{n \times n}$ is Schur stable iff there exists a diagonal matrix $P \in \mathbb{R}^{n \times n}, P>0$, such that $A^{\top} P A-P \prec$ 0 .

\section{DESIGN OF INTERVAL OBSERVER AND PREDICTOR}

In this section, we present new interval estimators (an IO and an IP) for system (1). Conditions of existence (given in the form of LMIs), for both IO and IP, are presented in Section III-A and III-B, respectively. The design of a static feedback controller for the IP is addressed in Section III-C. For clarity of exposition, the proofs of all results of this and the forthcoming sections will be presented in appendixes.

\section{A. Interval observer}

In this section, we investigate the design of an IO for (1) by exploiting the available measurement. To this end, let us evoke Assumption 1 and rewrite (1) as

$$
\begin{aligned}
x_{k+1}= & \left(A_{0}-L C\right) x_{k}+\sum_{i=1}^{\nu} \lambda_{i}(\theta) \Delta A_{i} x_{k}+L y_{k} \\
& +\left(B_{0}+\sum_{i=1}^{\nu} \lambda_{i}(\theta) \Delta B_{i}\right) u_{k}-L v_{k}+w_{k}
\end{aligned}
$$


for any $L \in \mathbb{R}^{n \times p}$. First, let us denote

$$
\Delta A_{+}=\sum_{i=1}^{\nu} \Delta A_{i}^{+}, \quad \Delta A_{-}=\sum_{i=1}^{\nu} \Delta A_{i}^{-}
$$

and, similarly, $\Delta B=\sum_{i=1}^{\nu} \Delta B_{i}^{+}$.

Then, under assumptions $1-2$ and Lemma 1 , we replace the uncertain terms in (5) by their interval bounds, which leads to the following IO:

$$
\begin{aligned}
\bar{x}_{k+1}= & \left(A_{0}-L_{o} C\right) \bar{x}_{k}+\Delta A_{+} \bar{x}_{k}^{+}+\Delta A_{-} \underline{x}_{k}^{-}+B_{0} u_{k} \\
& +\Delta B u_{k}^{+}+L_{o} y_{k}-L_{o}^{+} \underline{v}_{k}+L_{o}^{-} \bar{v}_{k}+\bar{w}_{k} \\
\underline{x}_{k+1}= & \left(A_{0}-L_{o} C\right) \underline{x}_{k}-\Delta A_{+} \underline{x}_{k}^{-}-\Delta A_{-} \bar{x}_{k}^{+}+B_{0} u_{k} \\
& -\Delta B u_{k}^{-}+L_{o} y_{k}-L_{o}^{+} \bar{v}_{k}+L_{o}^{-} \underline{v}_{k}+\underline{w}_{k}
\end{aligned}
$$

where $L_{o} \in \mathbb{R}^{n \times p}$ is the observer gain to be determined. The fulfillment of relation (2) follows the non-negativity of the estimation errors $\bar{e}_{k}=\bar{x}_{k}-x_{k}$ and $\underline{e}_{k}=x_{k}-\underline{x}_{k}$, the respective conditions are given in the following lemma:

Lemma 4: Let assumptions 1-2 be satisfied. Then, provided that $A_{0}-L_{o} C$ is non-negative, the estimation errors are non-negative, i.e., $\underline{e}_{k}, \bar{e}_{k} \geq 0$ for all $k>0$.

Now, it is needed to derive stability conditions for IO (6). First, let us denote $\chi_{k}=\operatorname{vec}\left(\bar{x}_{k}, \underline{x}_{k}\right)$ and rewrite (6) as

$$
\chi_{k+1}=\left(\mathcal{A}_{0}-\tilde{L}_{o} C_{1}\right) \chi_{k}+\mathcal{A}_{+} \chi_{k}^{+}+\mathcal{A}_{-} \chi_{k}^{-}+\delta_{k}
$$

where $\mathcal{A}_{0}=\operatorname{diag}\left(A_{0}, A_{0}\right) \in \mathbb{R}^{2 n \times 2 n}, \tilde{L}_{o}=\operatorname{diag}\left(L_{o}, L_{o}\right) \in$ $\mathbb{R}^{2 n \times 2 p} C_{1}=\operatorname{diag}(C, C) \in \mathbb{R}^{2 p \times 2 n}, \delta_{k}=\operatorname{vec}\left(\bar{\delta}_{k}, \underline{\delta}_{k}\right)$, and

$$
\begin{gathered}
\mathcal{A}_{+}=\left[\begin{array}{cc}
\Delta A_{+} & 0 \\
-\Delta A_{-} & 0
\end{array}\right], \quad \mathcal{A}_{-}=\left[\begin{array}{cc}
0 & \Delta A_{-} \\
0 & -\Delta A_{+}
\end{array}\right], \\
\bar{\delta}_{k}=B_{0} u_{k}+\Delta B u_{k}^{+}+L_{o} y_{k}-L_{o}^{+} \underline{v}_{k}+L_{o}^{-} \bar{v}_{k}+\bar{w}_{k}, \\
\underline{\delta}_{k}=B_{0} u_{k}-\Delta B u_{k}^{-}+L_{o} y_{k}-L_{o}^{+} \bar{v}_{k}+L_{o}^{-} \underline{v}_{k}+\underline{w}_{k} .
\end{gathered}
$$

For ease of notation in the sequel, let us denote $\tilde{U}=\operatorname{diag}(U, U)$ and $\tilde{P}=\operatorname{diag}(P, P)$ for some decision variables $P \in \mathbb{R}^{n \times n}$ and $U \in \mathbb{R}^{n \times p}$. A gain $L_{o}$ that stabilizes (6) and satisfies the restrictions of Lemma 4 can be computed by verifying the following conditions:

Theorem 1: Let assumptions $1-3$ be satisfied. If there exist diagonal matrices $\tilde{P}, Q_{1}, Q_{2}, Q_{3}, \Omega_{+}, \Omega_{-}, \Psi \in \mathbb{R}^{2 n \times 2 n}$, matrices $\Gamma \in \mathbb{R}^{2 n \times 2 n}$ and $\tilde{U} \in \mathbb{R}^{2 n \times p}$, such that the following LMIs are verified:

$$
\begin{array}{ccccc}
\multicolumn{8}{c}{\tilde{P} \mathcal{A}_{0}-\tilde{U} C_{1} \geq 0} \\
{\left[\begin{array}{ccccc}
\tilde{P}-Q_{1} & -\Omega_{+} & -\Omega_{-} & 0 & \mathcal{A}_{0}^{\top} \tilde{P}-C_{1}^{\top} \tilde{U}^{\top} \\
\star & -Q_{2} & -\Psi & 0 & \mathcal{A}_{+}^{\top} \tilde{P} \\
\star & \star & -Q_{3} & 0 & \mathcal{A}_{\bar{P}}^{\dagger} \tilde{P} \\
\star & \star & \star & \Gamma & \tilde{P} \\
\star & \star & \star & \star & \tilde{P}
\end{array}\right] \succeq 0} \\
\tilde{P}>0, & \Gamma \succ 0, & Q_{1}, Q_{2}, Q_{3}, \Omega_{+}, \Omega_{-} \geq 0 \\
Q_{1}+\min \left\{Q_{2}, Q_{3}\right\}+2 \min \left\{\Omega_{+}, \Omega_{-}\right\}>0
\end{array}
$$

then system (6) with a gain $L_{o}=P^{-1} U$ is an IO for system (1), i.e., relation (2) is satisfied and, in addition, $\chi \in \ell_{\infty}^{2 n}$ provided that $\delta \in \ell_{\infty}^{2 n}$.

Remark 1: Depending on the pair $\left(A_{0}, C\right)$, there might be no $L_{o}$ that satisfies the conditions of Theorem 1 , where the first one ensures non-negativity of $D_{o}=A_{0}-L_{o} C$, and the rest provide boundedness of $\chi_{k}$. Nevertheless, the first requirement may be alleviated by introducing a cooperative change of coordinates:

Theorem 3: [20] Let assumptions 1-3 be satisfied, and there exist a matrix $R \in \mathbb{R}_{+}^{n \times n}$ having the same eigenvalues as $D_{o}$, and the pairs $\left(D_{o}, e_{1}\right)$ and $\left(R, e_{2}\right)$, which are observable for some $e_{1} \in \mathbb{R}^{1 \times n}$, $e_{2} \in \mathbb{R}^{1 \times n}$. Then, the relation (2) is satisfied for

$$
\begin{aligned}
\underline{x}_{k} & =S^{+} \underline{\xi}_{k}-S^{-} \bar{\xi}_{k}, \quad \bar{x}_{k}=S^{+} \bar{\xi}_{k}-S^{-} \underline{\xi}_{k} \\
\bar{\xi}_{k+1} & =R \bar{\xi}_{k}+F y_{k}-F^{+} \underline{v}_{k}+F^{-} \bar{v}_{k}+\left(S^{-1}\right)^{+} \bar{w}_{k}-\left(S^{-1}\right)^{-} \underline{w}_{k} \\
\underline{\xi}_{k+1} & =R \underline{\xi}_{k}+F y_{k}-F^{+} \bar{v}_{k}+F^{-} \underline{v}_{k}+\left(S^{-1}\right)^{+} \underline{w}_{k}-\left(S^{-1}\right)^{-} \bar{w}_{k} \\
\underline{\xi}_{0} & =\left(S^{-1} \underline{x}_{0}-\left(S^{-1}\right)^{-} \bar{x}_{0}, \quad \bar{\xi}_{0}=\left(S^{-1}\right) \bar{x}_{0}-\left(S^{-1}\right)^{-} \underline{x}_{0}\right.
\end{aligned}
$$

where $S=O_{R} O_{D_{o}}^{-1}\left(O_{D_{o}}\right.$ and $O_{R}$ are the observability matrices of the pairs $\left(D_{o}, e_{1}\right)$ and $\left(R, e_{2}\right)$, respectively), and $F=S^{-1} L_{o}$.

\section{B. Interval predictor}

As discussed in the previous subsection, IO (6) computes an interval $\left[\underline{x}_{k}, \bar{x}_{k}\right]$ in which the admissible values of $x_{k}$ are guaranteedly confined for all $k \in \mathbb{Z}_{+}$and all $\theta \in \Theta$. However, since it requires knowledge of $y_{k}$ (which is obviously unknown in future steps), this IO is unsuitable for prediction of the system behaviour.

Following an idea conceived for linear systems [17], this section addresses the design of an interval predictor: an estimator that satisfies relation (2), but that requires only information on the system dynamics, the bounds on the matrices $A$ and $B$ and the bounds on the disturbances.

To avoid confusion with the states of the IO, we denote $\bar{z}_{k}, \underline{z}_{k}$ as, respectively, the upper and lower predictive bounds of $x_{k}$, and $L_{p}$ as the predictor gain (which replaces $L$ in (5)). By definition, $L_{p}=L_{p}^{+}-L_{p}^{-} \in \mathbb{R}^{n \times p}$, for $L_{p}^{-}, L_{p}^{+} \in \mathbb{R}_{+}^{n \times p}$. Then, let us evoke Lemma 1 and Assumption 4 to write

$$
L_{p}^{+} C \underline{z}_{k}-L_{p}^{-} C \bar{z}_{k} \leq L_{p} C z_{k} \leq L_{p}^{+} C \bar{z}_{k}-L_{p}^{-} C \underline{z}_{k} .
$$

Hence, the relation above allows us to rewrite (6) by replacing the terms unavailable for prediction (i.e., $L y_{k}-L v_{k}+w_{k}=L C x_{k}+w_{k}$ ) with their respective bounds:

$$
\begin{aligned}
\bar{z}_{k+1}= & \left(A_{0}-L_{p} C\right) \bar{z}_{k}+\Delta A_{+} \bar{z}_{k}^{+}+\Delta A_{-} \underline{z}_{k}^{-}+L_{p}^{+} C \bar{z}_{k} \\
& -L_{p}^{-} C \underline{z}_{k}+B_{0} u_{k}+\Delta B u_{k}^{+}+\bar{w}_{k} \\
\underline{z}_{k+1}= & \left(A_{0}-L_{p} C\right) \underline{z}_{k}-\Delta A_{+} \underline{z}_{k}^{+}-\Delta A_{-} \bar{z}_{k}^{-}+L_{p}^{+} C \underline{z}_{k} \\
& -L_{p}^{-} C \bar{z}_{k}+B_{0} u_{k}-\Delta B u_{k}^{-}+\underline{w}_{k}
\end{aligned}
$$

Note that under assumptions $1-4$, the IP (10) is composed solely of known terms. The following lemma gives the condition for nonnegativity of the prediction errors $\bar{\epsilon}_{k}=\bar{z}_{k}-x_{k}$ and $\underline{\epsilon}_{k}=x_{k}-\underline{z}_{k}$ :

Lemma 5: Let assumptions $1-4$ be satisfied. Then, provided that $A_{0}-L_{p} C$ is non-negative, the prediction errors are non-negative, i.e., $\underline{\epsilon}_{k}, \bar{\epsilon}_{k} \geq 0$ for all $k \in \mathbb{Z}_{+}$.

Now, let us address the conditions for the stability of system (10). Since this system is nonlinear, we will derive these conditions by denoting $\mathcal{Z}_{k}=\operatorname{vec}\left(\bar{z}_{k}, \underline{z}_{k}\right)$, which allows us to rewrite (10) as:

$$
\mathcal{Z}_{k+1}=\left(\mathcal{A}_{0}+\tilde{L}_{p} C_{2}\right) \mathcal{Z}_{k}+\mathcal{A}_{+} \mathcal{Z}_{k}^{+}+\mathcal{A}_{-} \mathcal{Z}_{k}^{-}+\varrho_{k},
$$

where $\mathcal{A}_{0}, \mathcal{A}_{+}$and $\mathcal{A}_{-}$are the same as in (7), $\tilde{L}_{p}=$ $\operatorname{diag}\left(L_{p}^{-}, L_{p}^{-}\right) \in \mathbb{R}^{2 n \times 2 p}, \varrho_{k}=\operatorname{vec}\left(\bar{\varrho}_{k}, \underline{\varrho}_{k}\right)$ and

$$
\begin{gathered}
C_{2}=\left[\begin{array}{cc}
C & -C \\
-C & C
\end{array}\right], \\
\varrho_{k}=B_{0} u_{k}+\Delta B u_{k}^{+}+\bar{w}_{k}, \quad \underline{\varrho}_{k}=B_{0} u_{k}-\Delta B u_{k}^{-}+\underline{w}_{k} .
\end{gathered}
$$

The next theorem presents conditions that any gain $L_{p}$ has to fulfill in order to render IP (11) stable:

Theorem 2: Let assumptions $1-4$ be satisfied and let $L_{p}$ be a given gain. If there exist a matrix $\tilde{P}_{1} \in \mathbb{R}^{2 n \times 2 n}$, diagonal matrices $Q_{1}, Q_{2}, Q_{3}, \Omega_{+}, \Omega_{-}, \Psi, \Gamma \in \mathbb{R}^{2 n \times 2 n}$, such that the following linear 
matrix inequalities are verified:

$$
\begin{gathered}
{\left[\begin{array}{ccccc}
\tilde{P}_{1}-Q_{1} & -\Omega_{+} & -\Omega_{-} & 0 & \left(\tilde{P}_{1} \mathcal{A}_{0}+\tilde{P}_{1} \tilde{L}_{p} C_{2}\right)^{\top} \\
\star & -Q_{2} & -\Psi & 0 & \left(\tilde{P}_{1} \mathcal{A}_{+}\right)^{\top} \\
\star & \star & -Q_{3} & 0 & \left(\tilde{P}_{1} \mathcal{A}_{-}\right)^{\top} \\
\star & \star & \star & \Gamma & \tilde{P}_{1} \\
\star & \star & \star & \star & \tilde{P}_{1}
\end{array}\right] \succeq 0} \\
\tilde{P}_{1} \succ 0, \\
Q=Q_{1}+\min \left\{Q_{2}, Q_{3}\right\}+2 \min \left\{\Omega_{+}, \Omega_{-}\right\}>0,
\end{gathered}
$$

then system (11) is ISS with respect to the input $\varrho \in \ell_{\infty}^{2 n}$.

Finally, sufficient conditions for the existence of a gain $L_{p}$ providing non-negativity of the matrix $A_{0}-L_{p} C$ and stability of (11) simultaneously are obtained by the following corollary:

Corollary 1: Let assumptions $1-4$ be satisfied. If there exist diagonal matrices $\tilde{P}_{2}, Q_{1}, Q_{2}, Q_{3}, \Omega_{+}, \Omega_{-}, \Psi, \Gamma \in \mathbb{R}^{2 n \times 2 n}$ and $U^{+}, U^{-} \in \mathbb{R}^{n \times p}$ such that the following linear matrix inequalities are verified:

$$
\begin{aligned}
& \tilde{P}_{2} \mathcal{A}_{0}-\tilde{U}^{+} C_{1}+\tilde{U}^{-} C_{1} \geq 0 \\
& {\left[\begin{array}{ccccc}
\tilde{P}_{2}-Q_{1} & -\Omega_{+} & -\Omega_{-} & 0 & \left(\tilde{P}_{2} \mathcal{A}_{0}+\tilde{U}^{-} C_{2}\right)^{\top} \\
\star & -Q_{2} & -\Psi & 0 & \left(\tilde{P}_{2} \mathcal{A}_{+}\right)^{\top} \\
\star & \star & -Q_{3} & 0 & \left(\tilde{P}_{2} \mathcal{A}_{-}\right)^{\top} \\
\star & \star & \star & \Gamma & \tilde{P}_{2} \\
\star & \star & \star & \star & \tilde{P}_{2}
\end{array}\right] \succeq 0} \\
& Q_{1}, Q_{2}, Q_{3}, \Omega_{+}, \Omega_{-}, U^{+}, U^{-} \geq 0, \quad \Gamma \succ 0, \quad P_{2}>0 \\
& \tilde{P}_{2}=\operatorname{diag}\left(P_{2}, P_{2}\right), \quad \tilde{U}^{+}=\operatorname{diag}\left(U^{+}, U^{+}\right), \quad \tilde{U}^{-}=\operatorname{diag}\left(U^{-}, U^{-}\right) \text {, } \\
& Q=Q_{1}+\min \left\{Q_{2}, Q_{3}\right\}+2 \min \left\{\Omega_{+}, \Omega_{-}\right\}>0
\end{aligned}
$$

then system (10) with gains $L_{p}^{-}=P_{2}^{-1} U^{-}$and $L_{p}^{+}=P_{2}^{-1} U^{+}$is an IP for system (1), i.e., the relation $\underline{z}_{k} \leq x_{k} \leq \bar{z}_{k}$ is satisfied for all $k \in \mathbb{Z}_{+}$and the system (11) is ISS with respect to the input $\varrho \in \ell_{\infty}^{2 n}$.

\section{Control design}

In this section, we will address a feedback control design for the IP (10). To this end, the following simplifying assumption is needed:

Assumption 5: Let $\Delta B=0$.

Remark 2: Assumption 5 is imposed to streamline the presentation. Indeed, if system (1) is polytopic with $\Delta B \neq 0$, the design conditions given in the following are affine and have to be checked over all of its vertices. This scenario requires an intricated presentation, which we would like to avoid.

Then, if the control $u_{k}$ in (11) is selected such as

$$
u_{k}=K \mathcal{Z}_{k}+K_{+} \mathcal{Z}_{k}^{+}+K_{-} \mathcal{Z}_{k}^{-}+R \mathcal{W}_{k}
$$

where $\mathcal{W}_{k}=\operatorname{vec}\left(\bar{w}_{k}, \underline{w}_{k}\right)$ and for some $K, K_{-}, K_{+}, R \in \mathbb{R}^{m \times 2 n}$, the resulting dynamics is given by

$$
\mathcal{Z}_{k+1}=\mathcal{K} \mathcal{Z}_{k}+\mathcal{K}_{+} \mathcal{Z}_{k}^{+}+\mathcal{K}_{-} \mathcal{Z}_{k}^{-}+\tilde{D} \mathcal{W}_{k}
$$

where $\tilde{D}=\mathbb{I}_{2 n}+\mathcal{B}_{0} R, \mathcal{K}=\mathcal{A}_{0}+\tilde{L}_{p} C_{2}+\mathcal{B}_{0} K, \mathcal{K}_{+}=\mathcal{A}_{+}+\mathcal{B}_{0} K_{+}$ and $\mathcal{K}_{-}=\mathcal{A}_{-}+\mathcal{B}_{0} K_{-}$, in which, for ease of notation, we denote $\mathcal{B}_{0}=\left[B_{0}^{\top}, B_{0}^{\top}\right]^{\top}$. This brings us to the following result:

Theorem 4: Let assumptions $1-5$ be satisfied. If there exist matrices $P, Q_{1}, Q_{2}, Q_{3}, \Gamma, \Omega_{+}, \Omega_{-}, \Psi \in \mathbb{R}^{2 n \times 2 n}$ and $W_{1}, W_{2}, W_{3}, W_{4} \in \mathbb{R}^{m \times 2 n}$ such that the following inequalities are verified

$$
\begin{aligned}
& {\left[\begin{array}{ccccc}
P-Q_{1} & -\Omega_{+} & -\Omega_{-} & 0 & W_{1}^{\top} \mathcal{B}_{0}^{\top}+P D_{z}^{\top} \\
\star & -Q_{2} & -\Psi & 0 & W_{2}^{\top} \mathcal{B}_{0}^{\top}+P \mathcal{A}_{\neq}^{\top} \\
\star & \star & -Q_{3} & 0 & W_{3}^{\top} \mathcal{B}_{0}^{\top}+P \mathcal{A}_{-}^{\dagger} \\
\star & \star & \star & \Gamma & W_{4}^{\top} \mathcal{B}_{0}^{\top}+P \\
\star & \star & \star & \star & P
\end{array}\right] \succ 0} \\
& P>0, \quad \Gamma>0, \quad Q_{1}, Q_{2}, Q_{3}, \Omega_{+}, \Omega_{-} \geq 0, \\
& Q=Q_{1}+\min \left\{Q_{2}, Q_{3}\right\}+2 \min \left\{\Omega_{+}, \Omega_{-}\right\}>0 \text {, }
\end{aligned}
$$

then IP (11) under control (13) with gains $K=W_{1} P^{-1}, K_{+}=$ $W_{2} P^{-1}, K_{-}=W_{3} P^{-1}, R=W_{4} P^{-1}$ is ISS with respect to the inputs $\mathcal{W} \in \ell_{\infty}^{2 n}$.

\section{IO-MPC DESIGN}

In this section, we present the robust output feedback MPC scheme with guaranteed constraint satisfaction, based on the interval estimators introduced previously. Following the classic axioms of Mayne et al. [3], Section IV-A presents the stabilizing ingredients, while Section IV-B formulates the proposed algorithm and the main result concerning its properties.

\section{A. Stabilizing ingredients}

As a consequence of the ISS property depicted in Theorem 4 we have that, for $\tilde{\Gamma}=P^{-1} \Gamma P^{-1}$, the ellipsoid

$$
\tilde{\mathbb{X}}=\left\{x \in \mathbb{R}^{2 n}: x^{\top} P^{-1} x \leq \alpha^{-1} \sup _{k \geq 0} \mathcal{W}_{k}^{\top} \tilde{\Gamma} \mathcal{W}_{k}\right\}
$$

for $\alpha>0$ given in the proof of Theorem 4 (see the Appendix) is an invariant set for (14) and thus can be used as the terminal set. Consequently, the terminal cost weighting can be readily selected as $P^{-1}$ (i.e., the Lyapunov matrix used in Theorem 4). However, for well-posedness of the MPC scheme, the following assumption (which is conventional in MPC) is imposed:

Assumption 6: Let $\mathbb{X}_{f} \times \mathbb{X}_{f} \subseteq \tilde{\mathbb{X}} \subseteq \mathbb{X} \times \mathbb{X}$ and, the control input computed by (13) satisfy $u_{k} \in \mathbb{U}$ provided that $\mathcal{Z}_{k} \in \mathbb{X}_{f} \times \mathbb{X}_{f}$.

If the set $\mathbb{U}$ is ellipsoidal, one may relax Assumption 6 by introducing additional LMIs to Theorem 4:

Corollary 2: Let there exist symmetric and positive definite matrices $S \in \mathbb{R}^{m \times m}$ and $Z \in \mathbb{R}^{2 n \times 2 n}$ such that $\mathbb{U}=\left\{u \in \mathbb{R}^{m}\right.$ : $\left.u^{\top} S u \leq 1\right\}$ and $\mathcal{W}_{k} \in\left\{\mathcal{W} \in \mathbb{R}^{2 n}: \mathcal{W}^{\top} Z \mathcal{W} \leq 1\right\}$, and the conditions of Theorem 4 be satisfied with additional inequalities:

$$
\begin{aligned}
& \frac{\eta}{\alpha \kappa} \Gamma \leq \min \left\{\kappa^{-1} Z, P\right\}, P \geq \kappa Z^{-1}, \\
& {\left[\begin{array}{cccc}
\frac{\eta}{3} P & 0 & 0 & W_{1}^{\top}+W_{2}^{\top} \\
0 & \frac{\eta}{3} P & 0 & W_{3}^{\top}-W_{1}^{\top} \\
0 & 0 & \frac{\kappa}{3} P & W_{4}^{\top} \\
W_{1}+W_{2} & W_{3}-W_{1} & W_{4} & S^{-1}
\end{array}\right] \geq 0}
\end{aligned}
$$

for some constants $\eta>0$ and $\kappa>0$, then control (13) satisfies the constraint $u_{k} \in \mathbb{U}$ for all $\mathcal{Z}_{k} \in \mathbb{X}_{f} \times \mathbb{X}_{f}$.

Therefore, since a relation between $u_{k}$ and the set $\tilde{\mathbb{X}}$ (and, consequently, also $\mathbb{X}_{f}$ ) is established, the stage costs weighting the control input can also be selected as a function of $P^{-1}$. This fact will be used to show ISS in $\mathbb{X}_{f}$ of (1) with MPC in the next section.

In the following, we denote by $\underline{z}_{k, i}, \bar{z}_{k, i}$ the predictions obtained on the $i$-th step at the decision instant $k$, since we will reinitialize the predictor regularly. Previously we used the notation $\underline{z}_{k}, \bar{z}_{k}$, and the predictor in Section III was initialized just once at $k=0$.

\section{B. Design of the predictive controller}

The main idea of the proposed MPC scheme is as follows. Since the IP (10) depends solely on known variables, we can use it to predict an envelope of all trajectories of system (1) for any $\theta_{k} \in \Theta$, and use this prediction to verify constraint satisfaction. By running the IO (6), the information on the set-membership of the states of system (1) is updated with every new measurement $y_{k}$.

Let us define

$$
\overline{\hat{x}}_{k}=\min \left\{\bar{x}_{k}, \bar{z}_{k-1,1}\right\}, \quad \underline{\hat{x}}_{k}=\max \left\{\underline{x}_{k}, \underline{z}_{k-1,1}\right\}
$$

and, at each decision instant $k \in \mathbb{Z}_{+}$, we will initialize the IP (10) with $\underline{z}_{k, 0}=\underline{\hat{x}}_{k}$, and $\bar{z}_{k, 0}=\hat{\bar{x}}_{k}$. Thus, having an input sequence $\mathcal{S}_{N}=\left\{s_{0}, \ldots, s_{N-1}\right\}$ with $s_{i} \in \mathbb{U}$, we calculate the values of 
$\underline{z}_{k, i+1}, \bar{z}_{k, i+1}$ for $i=0, \ldots, N-1$ (under substitution of $u_{k+i}=s_{i}$ and using the fact that $\underline{w}_{k}, \bar{w}_{k}$ are given for all $k \in \mathbb{Z}_{+}$according to Assumption 3). Then, the Optimal Control Problem (OCP) solved by the MPC is stated as follows:

$$
\mathcal{S}_{N}^{k}:=\arg \min _{\mathcal{S}_{N}} V_{N}\left(\mathcal{Z}_{k, 0}, \ldots, \mathcal{Z}_{k, N}, \mathcal{S}_{N}\right)
$$

subject to the following constraints:

$$
\begin{gathered}
\underline{z}_{k, 0}=\underline{\hat{x}}_{k}, \quad \bar{z}_{k, 0}=\overline{\hat{x}}_{k} \\
\mathcal{Z}_{k, i+1} \text { computed by }(11) \\
\mathcal{Z}_{k, i+1} \subset \mathbb{X} \times \mathbb{X}, \quad s_{i} \subset \mathbb{U}, \\
\mathcal{Z}_{k, N} \in \mathbb{X}_{f} \times \mathbb{X}_{f}
\end{gathered}
$$

with a (quadratic) cost function $V_{N}$ defined by

$$
V_{N}\left(\mathcal{Z}_{k, 0}, \ldots, \mathcal{Z}_{k, N}, \mathcal{S}_{N}\right)=V_{f}\left(\mathcal{Z}_{k, N}\right)+\sum_{i=0}^{N-1} \ell\left(\mathcal{Z}_{k, i}, s_{i}\right)
$$

where $V_{f}(\mathcal{Z})=\mathcal{Z}^{\top} \Psi_{1} \mathcal{Z}, \quad \ell(\mathcal{Z}, s)=\mathcal{Z}^{\top} \Psi_{2} \mathcal{Z}+s^{\top} \Psi_{3} s$ with $\Psi_{1}, \Psi_{2}, \Psi_{3}$ being positive-definite symmetric weighting matrices. The algorithm below summarizes the MPC routine. The next theorem states the main result of this section.

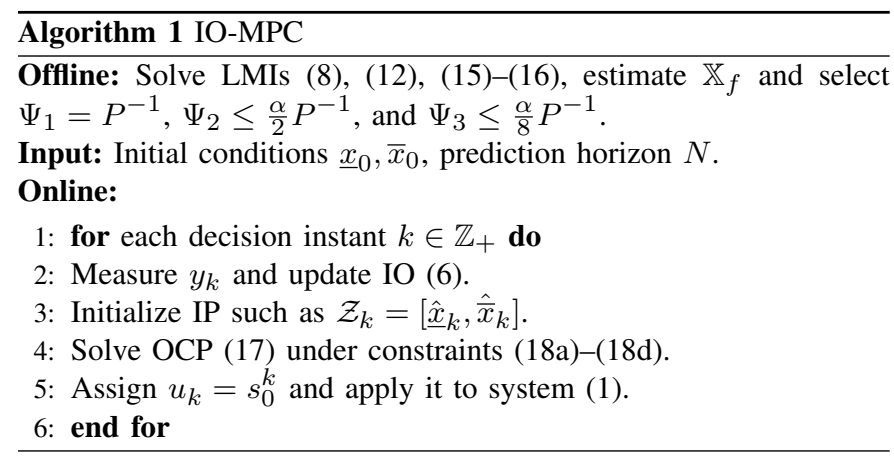

Theorem 5: Let $\left[\underline{x}_{0}, \bar{x}_{0}\right] \subset \mathbb{X}$ and assumptions $1-6$ be satisfied with $\left[\underline{w}_{k+1}, \bar{w}_{k+1}\right] \subseteq\left[\underline{w}_{k}, \bar{w}_{k}\right]$ for all $k \in \mathbb{Z}_{+}$. Then, following Algorithm 1, the closed-loop system composed by (1), (6) and (10) has the following features:

1) Recursive feasibility of reaching the terminal set in $N$ steps;

2) ISS of dynamics (11) in $\mathbb{X}_{f}$ and practical ISS for (1);

3) Constraint satisfaction.

\section{Complexity and contrast with the existing literature}

The complexity of solving Algorithm 1 scales linearly with $O(N n)$. Indeed, assuming that the number of hyperplanes needed to define sets $\mathbb{X}, \mathbb{U}$ and $\mathbb{X}_{f}$ depends linearly on $n$ and $m=n$, the (worst-case) number of variables describing constraints (18a)-(18d) is $10 N n$.

Note that this complexity is fixed, which is an interesting feature of the proposed method. Approaches using zonotopic estimation often require extra procedures to limit their increasing complexity, as well as real-time knowledge on the scheduling parameter [23].

Techniques such as presented in [15] [16], that also assume no measurement of $\theta_{k}$ fix a prediction horizon $N=1$ and impose a minmax optimization problem accounting for all vertices of the polytopic system, aiming to obtain a robust prediction. This implementation requires several relaxations for numerical tractability and the price is an increased number of variables and conservativeness.

Remark 3: Due to the terms $\mathcal{Z}^{+}, \mathcal{Z}^{-}$in (11), the OCP (17) is not a quadratic optimization problem by definition. However, since the epigraph of function $\max (\cdot)$ is convex over the Euclidean space,
(17) can be easily rewritten as a convex problem that can be solved efficiently (see Chapter 4.2 in [24]).

Remark 4: Note that $\mathcal{Z}_{k}^{-}$and $\mathcal{Z}_{k}^{+}$in (11) are piece-wise linear. Therefore, the set $\mathbb{X} \times \mathbb{X}$ can be decomposed on hypercubes, where (11) has an LTI representation. Hence, ideas concerning switched MPC (such as presented in [25]) could be implemented. This extension is skipped for brevity.

\section{NUMERICAL EXAMPLE}

In this section, we present a numerical example to illustrate the usefulness of the proposed MPC scheme. Consider the following LPV system:

$$
\begin{aligned}
x_{k+1} & =\left[\begin{array}{cc}
0.5 & 0.6+\theta_{k} \\
\theta_{k} & 0.3
\end{array}\right] x_{k}+\left[\begin{array}{l}
0 \\
1
\end{array}\right] u_{k}+w_{k} \\
y_{k} & =\left[\begin{array}{ll}
0 & 1
\end{array}\right] x_{k}+v_{k}
\end{aligned}
$$

where $x_{k}=\operatorname{vec}\left(x_{1}, x_{2}\right) \in \mathbb{R}^{2}, \theta_{k} \in \Theta=[-0.1,0.1], w_{k} \in$ $[-0.1,0.1] \times[-0.1,0.1]$, and $v_{k} \in[-0.1,0.1]$. The constraint sets are defined as $\mathbb{X}=[3,-12] \times[3,-12]$ and $\mathbb{U}=[-2,2]$. Clearly, this system can be rewritten as (1) with the matrices

$$
A_{0}=\left[\begin{array}{cc}
0.5 & 0.6 \\
0 & 0.3
\end{array}\right], A(\theta)=\left[\begin{array}{ll}
0 & \theta \\
\theta & 0
\end{array}\right] \text {. }
$$

and interpolating functions $\lambda_{1}=\frac{\theta-\underline{\theta}}{\bar{\theta}-\theta}$ and $\lambda_{2}=\frac{\bar{\theta}-\theta}{\bar{\theta}-\theta}$. We considered initial conditions for the IP/IO as $\overline{\bar{x}}_{0}=\operatorname{vec}(-7,-12)$ and $\underline{x}_{0}=$ $\operatorname{vec}(-6,-10)$ and several initial conditions for (19) satisfying $x_{0} \in$ $\left[\underline{x}_{0}, \bar{x}_{0}\right]$. We ran 100 simulations of this scenario, each with a time span of $T=20$ steps, considering several realizations of $\theta_{k}, w_{k}$, and $v_{k}$.

The gains for the IP and the IO, obtained by solving the offline LMIs of Section III, are $L_{o}=[0.489,0.1945]$ and $L_{p}=$ $[0.232,0.122]$. For the MPC algorithm, we solve the conditions of Theorem 4 to obtain $\alpha=1.107$ and

$$
P=\left[\begin{array}{cc}
1.79 & \star \\
-0.285 & 1.150
\end{array}\right], \quad \Gamma=\left[\begin{array}{cc}
11.57 & \star \\
-1.358 & 3.63
\end{array}\right],
$$

and thus we can estimate $\mathbb{X}_{f}$ and select $\Psi_{1}, \Psi_{2}$ and $\Psi_{3}$ accordingly. Finally, we select the prediction horizon as $N=10$.

Fig. 1 illustrates the trajectories of system (19) and the IP (10), in contrast to the constraint set. It is worth noticing that all constraints were respected, including those on the unmeasured state. The IP even reaches the boundary of constraint, indicating low conservativeness.

The control input computed by solving OCP (17) also fulfilled the constraints, as shown in Fig. 2. Finally, Fig. 3 shows the estimated feasible regions for the initial conditions of IP (10). We did not observe any improvement after $N=10$. The mean computation time for solving OCP (17) was $0.22 \pm 0.0313$ second/step, with a maximum of 0.7725 second.

All simulations were performed using MATLAB 2017a, with an Intel i7-8565U processor (1.8GHz) and 16GB RAM. Also, we used YALMIP [26] to solve the offline LMIs and to solve the optimization problem (using an interior-point method provided by the fmincon solver).

\section{CONCLUSIONS}

In this paper, we have presented a novel robust, output feedback MPC for LPV systems, guaranteeing recursive stability and constraint satisfaction. By adding an interval observer and a predictor on the algorithm, the obtained MPC is similar to a conventional MPC in the sense that it requires the same stabilizing ingredients. The interval estimators, as well as the stabilizing features for the MPC, are 

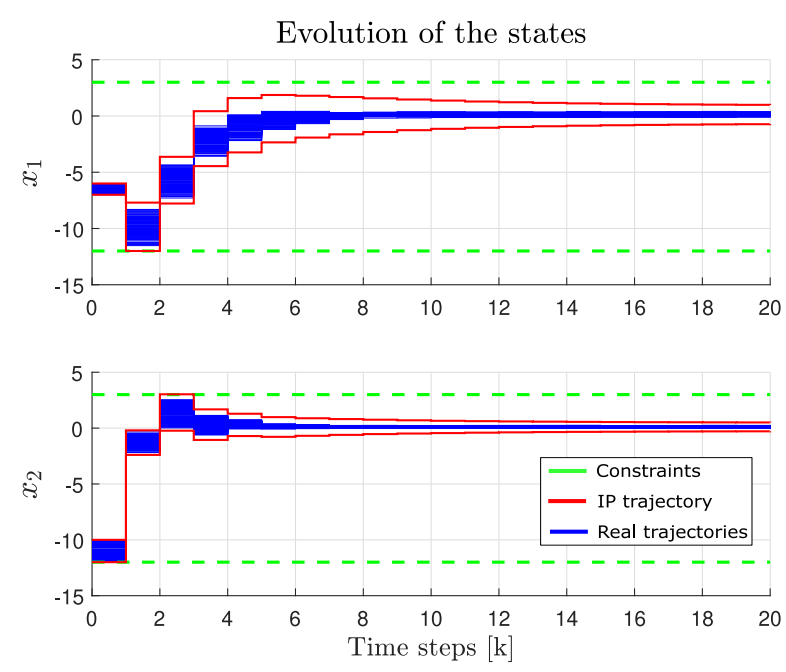

Fig. 1. Trajectories of (19) and (11) in contrast to the constraint set.

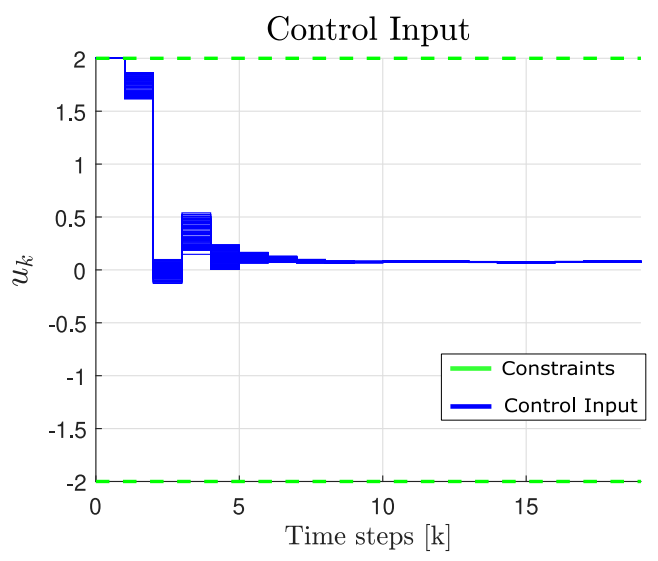

Fig. 2. Evolution of the control inputs

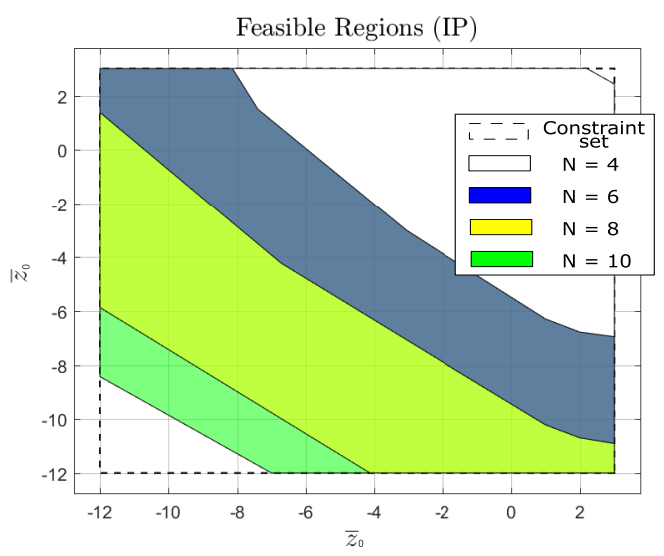

Fig. 3. Comparison of the feasible regions for prediction horizon with different lengths $(N \in\{\mathbf{4}, \mathbf{6}, \mathbf{8}, \mathbf{1 0}\})$. These regions were computed using YALMIP [26] and MPT [27]. obtained through the offline solution of LMIs. A numerical example was proposed to illustrate the usefulness of the methodology.

Future research includes the case of time-delayed systems and using real-time measurement of the scheduling parameter to enhance the proposed algorithm.

\section{APPENDIX \\ PROOF OF LEMMA 4}

First, by applying (4) in $\lambda_{i} x_{k}$, we obtain

$$
\begin{aligned}
& \underline{\lambda}_{i}^{+} \underline{x}_{k}^{+}-\bar{\lambda}_{i}^{+} \underline{x}_{k}^{-}-\underline{\lambda}_{i}^{-} \bar{x}_{k}^{+}+\bar{\lambda}_{i}^{-} \bar{x}_{k}^{-} \leq \lambda_{i} x_{k} \leq \\
& \bar{\lambda}_{i}^{+} \bar{x}_{k}^{+}-\underline{\lambda}_{i}^{+} \bar{x}_{k}^{-}-\bar{\lambda}_{i}^{-} \underline{x}_{k}^{+}+\underline{\lambda}_{i}^{-} \underline{x}_{k}^{-} .
\end{aligned}
$$

Since $\lambda_{i} \in[0,1], \bar{\lambda}_{i}^{+}=1$ and $\underline{\lambda}_{i}^{+}=\bar{\lambda}_{i}^{-}=\underline{\lambda}_{i}^{-}=0$, by definition. Under this, we have that the relation $-\underline{x}_{k}^{-} \leq \lambda_{i}\left(\theta_{k}\right) x_{k} \leq \bar{x}_{k}^{+}$holds. Then, since the vertices of the polytopic system are known, we apply (3) to obtain the following relation

$$
-\Delta A_{+} \underline{x}_{k}^{-}-\Delta A_{-} \bar{x}_{k}^{+} \leq \sum_{i=1}^{N} \lambda_{i}\left(\theta_{k}\right) \Delta A_{i} x_{k} \leq \Delta A_{+} \bar{x}_{k}^{+}+\Delta A_{-} \underline{x}_{k}^{-}
$$

Finally, the same idea applies to the term proportional to $u_{k}$, leading to the following relation:

$$
-\Delta B_{i} u_{k}^{-} \leq-\left(\Delta B_{i} u_{k}\right)^{-} \leq \lambda_{i} \Delta B_{i} u_{k} \leq\left(\Delta B_{i} u_{k}\right)^{+} \leq \Delta B_{i} u_{k}^{+} .
$$

Now, computing the increments of the estimation errors $\bar{e}_{k}, \underline{e}_{k}$ and taking (20)-(21) into account, we have

$$
\bar{e}_{k+1}=D_{o} \bar{e}_{k}+r_{1,1}+r_{1,2}, \quad \underline{e}_{k+1}=D_{o} \underline{e}_{k}+r_{2,1}+r_{2,2}
$$

where $D_{o}=A_{0}-L_{o} C$,

$$
\begin{aligned}
r_{1,1}= & \Delta A_{+} \bar{x}_{k}^{+}+\Delta A_{-} \underline{x}_{k}^{-}-\sum_{i=1}^{N} \lambda_{i}\left(\theta_{k}\right) \Delta A_{i} x_{k}+\Delta B u_{k}^{+} \\
& -\sum_{i=1}^{N} \lambda_{i}\left(\theta_{k}\right) \Delta B_{i} u_{k} \\
r_{1,2}= & L_{o} v_{k}-L_{o}^{+} \underline{v}_{k}+L_{o}^{-} \bar{v}_{k}+\bar{w}_{k}-w_{k} \\
r_{2,1}= & \sum_{i=1}^{N} \lambda_{i}\left(\theta_{k}\right) \Delta A_{i} x_{k}-\left(-\Delta A_{+} \underline{x}_{k}^{-}-\Delta A_{-} \bar{x}_{k}^{+}\right) \\
& +\sum_{i=1}^{N} \lambda\left(\theta_{k}\right) \Delta B_{i} u_{k}+\Delta B u_{k}^{-} \\
r_{2,2}= & L_{o}^{+} \bar{v}_{k}-L_{o}^{-} \underline{v}_{k}-L_{o} v_{k}+w_{k}-\underline{w}_{k} .
\end{aligned}
$$

From assumptions 1-3 and relations (20)-(21), we have that quantities (22) are positive. Hence, if $D_{o}$ is non-negative, we have that $\bar{e}_{k}, \underline{e}_{k}>0$ for all $k>0$ by Lemma 2 , and thus satisfying relation (2).

\section{PROOF OF THEOREM 1}

Let us consider a Lyapunov function candidate $V_{k}=\chi_{k}^{\top} \tilde{P} \chi_{k}$, whose increments are given by

$$
\begin{aligned}
V_{k+1}-V_{k}= & \zeta_{k}^{\top} \Sigma \zeta_{k}-\chi_{k}^{\top} Q_{1} \chi_{k}-\chi_{k}^{+\top} Q_{2} \chi_{k}^{+}-\chi_{k}^{-\top} Q_{3} \chi_{k}^{-} \\
& -2 \chi_{k}^{\top} \Omega_{+} \chi_{k}^{+}-2 \chi_{k}^{\top} \Omega_{-} \chi_{k}^{-}-2 \chi_{k}^{+\top} \Psi \chi_{k}^{-}+\delta_{k}^{\top} \Gamma \delta_{k},
\end{aligned}
$$

where $\zeta_{k}=\operatorname{vec}\left(\chi_{k}, \chi_{k}^{+}, \chi_{k}^{-}, \delta_{k}\right)$ and

$\Sigma=\left[\begin{array}{cccc}\mathcal{D}_{o}^{\top} \tilde{P} \mathcal{D}_{o}-\tilde{P}+Q_{1} & \mathcal{D}_{o}^{\top} \tilde{P} \mathcal{A}_{+}+\Omega_{+} & \mathcal{D}_{o}^{\top} \tilde{P} \mathcal{A}_{-}+\Omega_{-} & \mathcal{D}_{o}^{\top} \tilde{P} \\ \star & \mathcal{A}_{+}^{\top} \tilde{P} \mathcal{A}_{+}+Q_{2} & \mathcal{A}_{+}^{\top} \tilde{P} \mathcal{A}_{-}+\Psi & \mathcal{A}_{\neq}^{\top} \tilde{P} \\ \star & \star & \mathcal{A}_{-}^{\top} \tilde{P} \mathcal{A}_{-}+Q_{3} & \mathcal{A}_{-}^{\dagger} \tilde{P} \\ \star & \star & \star & \tilde{P}-\Gamma\end{array}\right]$

and, for brevity, $\mathcal{D}_{o}=\mathcal{A}_{0}-\tilde{L}_{o} C_{1}$.

If $Q=Q_{1}+\min \left\{Q_{2}, Q_{3}\right\}+2 \min \left\{\Omega_{+}, \Omega_{-}\right\} \succ 0, \Gamma \succ 0$ and $\Sigma \preccurlyeq 0$ and provided that $\delta \in \mathcal{L}_{\infty}^{2 n}$, then the stated stability conditions are fulfilled and system (6) is ISS with respect to the input $\delta_{k}$ (the 
diagonal matrix $\Psi$ can be sign indefinite since $\chi_{k}^{+\top} \Psi \chi_{k}^{-}=0$ by definition):

$$
V_{k+1}-V_{k}=-\chi_{k}^{\top} Q \chi_{k}+\delta_{k}^{\top} \Gamma \delta_{k}
$$

Hence, it is needed to show that the above stability conditions can be formulated as LMIs. First, since $\tilde{P}>0$, we can decompose $\Sigma$ as

$$
\Sigma=\left[\begin{array}{c}
\mathcal{D}_{o}^{\top} \tilde{P} \\
\mathcal{A}_{\neq}^{\top} \tilde{P} \\
\mathcal{A}_{\tilde{\tilde{P}}}^{\dagger} \tilde{P} \\
\tilde{\tilde{P}}
\end{array}\right] \tilde{P}^{-1}\left[\begin{array}{c}
\tilde{P} \mathcal{D}_{o} \\
\tilde{P} \mathcal{A}_{+} \\
\tilde{P} \mathcal{A}_{-} \\
\tilde{P}
\end{array}\right]^{\top}-\left[\begin{array}{cccc}
\tilde{P}-Q_{1} & -\Omega_{+} & -\Omega_{-} & 0 \\
-\Omega_{+} & -Q_{2} & -\Psi & 0 \\
-\Omega_{-} & -\Psi & -Q_{3} & 0 \\
0 & 0 & 0 & \Gamma
\end{array}\right]
$$

then, by applying the Schur complement, the condition $\Sigma \preceq 0$ can be equivalently written as

$$
\left[\begin{array}{ccccc}
\tilde{P}-Q_{1} & -\Omega_{+} & -\Omega_{-} & 0 & \mathcal{D}_{o}^{\top} \tilde{P} \\
\star & -Q_{2} & -\Psi & 0 & \mathcal{A}_{\neq}^{\top} \tilde{P} \\
\star & \star & -Q_{3} & 0 & \mathcal{A}_{-}^{\dagger} \tilde{P} \\
\star & \star & \star & \Gamma & \tilde{P} \\
\star & \star & \star & \star & \tilde{P}
\end{array}\right] \succeq 0
$$

Let us denote $U=P L_{o} \in \mathbb{R}_{+}^{n \times p}$. Recalling that $P D_{o}=P A_{0}-P L_{o} C$, then inequality (23) becomes linear in $P, Q_{1}, Q_{2}, Q_{3}, \Gamma, \Omega_{+}, \Omega_{-}, \Psi$ and $U$ :

$$
\left[\begin{array}{cccc}
\tilde{P}-Q_{1} & -\Omega_{+} & -\Omega_{-} & 0 \\
\star & -Q_{2} & -\Psi & 0 \\
\star & \star & -Q_{3} & 0 \\
\star & \star & \star & \Gamma \\
\star & \star & \star & \star
\end{array}\right.
$$

$$
\left.\begin{array}{c}
\mathcal{A}_{0}^{\top} \tilde{P}-C_{1}^{\top} \tilde{U}^{\top} \\
\mathcal{A}_{\neq}^{\top} \tilde{P} \\
\mathcal{A}_{\tilde{\tilde{P}}}^{\dagger} \tilde{P} \\
\tilde{P}
\end{array}\right] \succeq 0
$$

Finally, since $\tilde{P}>0$ and diagonal, the constraint $A_{0}-L_{o} C \geq 0$ follows from $\tilde{P} \mathcal{A}_{0}-\tilde{U} C_{1} \geq 0$, which is also linear in $\tilde{P}$ and $\tilde{U}$, finalizing the proof.

\section{PROOF OF LEMMA 5}

Analogously to Lemma 4, let us compute increments of the estimation errors $\underline{\epsilon}_{k}, \bar{\epsilon}_{k}$ :

$$
\begin{aligned}
\bar{\epsilon}_{k+1} & =\left(A_{0}-L_{p} C\right) \bar{\epsilon}_{k}+r_{1,1}+\tilde{r}_{1,2}, \\
\underline{\epsilon}_{k+1} & =\left(A_{0}-L_{p} C\right) \underline{\epsilon}_{k}+r_{2,1}+\tilde{r}_{2,2}
\end{aligned}
$$

where $r_{1,1}$ and $r_{2,1}$ are as in (22) and

$$
\begin{aligned}
& \tilde{r}_{1,2}=L_{p}^{+} C \bar{z}_{k}-L_{p}^{-} C \underline{z}_{k}-L_{p} C z_{k}+\bar{w}_{k}-w_{k} \\
& \tilde{r}_{2,2}=L_{p} C z_{k}-L_{p}^{+} C \underline{z}_{k}+L_{p}^{-} C \bar{z}_{k}+w_{k}-\underline{w}_{k} .
\end{aligned}
$$

Then, according to relations (20), (21) and (9), all inputs (the terms independent on $\underline{\epsilon}$ and $\bar{\epsilon}$ ) in (24) are non-negative. Thus, if $A_{0}-L_{p} C$ is also non-negative, we have that $\bar{\epsilon}_{k}, \underline{\epsilon}_{k} \geq 0$ for all $k \in \mathbb{Z}_{+}$by Lemma 2.

\section{PROOF OF THEOREM 2}

The proof follows the same rationale as in Theorem 1 and is only sketched. By considering a Lyapunov function candidate given by $V_{k}=\mathcal{Z}_{k}^{\top} \tilde{P}_{1} \mathcal{Z}_{k}$, one can show input-to-state stability, i.e.,

$$
V_{k+1}-V_{k}=-\mathcal{Z}_{k}^{\top} Q \mathcal{Z}_{k}+\varrho_{k}^{\top} \Gamma \varrho_{k}
$$

and the LMI conditions are stated by applying the Schur complement in the resulting stability conditions.

\section{PROOF OF COROLLARY 1}

This proof follows directly from the implications of Lemma 5 and Theorem 2. Indeed, evoking [20], the first constraint makes $A_{0}-L_{p} C$ non-negative, thus satisfying the conditions on cooperativity imposed on Lemma 5. Then, by introducing a new variable $U=P_{2} L_{p}$ and recalling that $U=U^{+}-U^{-}$by definition, this search for $L_{p}$ is additionally constrained by the conditions stated in Theorem 2 . This guarantees stability of (10), concluding the proof.

\section{PROOF OF THEOREM 4}

Let us consider a Lyapunov function candidate given by $V_{k}=$ $\mathcal{Z}_{k}^{\top} P^{-1} \mathcal{Z}_{k}$, whose increments can be written as

$$
\begin{aligned}
V_{k+1}-V_{k}=\tilde{\zeta}_{k}^{\top} \tilde{\Pi} \tilde{\zeta}_{k}-\mathcal{Z}_{k}^{\top} \tilde{Q}_{1} \mathcal{Z}_{k}-\mathcal{Z}_{k}^{+\top} \tilde{Q}_{2} \mathcal{Z}_{k}^{+}-\mathcal{Z}_{k}^{-\top} \tilde{Q}_{3} \mathcal{Z}_{k}^{-} \\
+\mathcal{W}_{k}^{\top} \tilde{\Gamma} \mathcal{W}_{k}-2 \mathcal{Z}_{k}^{\top} \tilde{\Omega}_{+} \mathcal{Z}_{k}^{+}-2 \mathcal{Z}_{k}^{\top} \tilde{\Omega}_{-} \mathcal{Z}_{k}^{-}-2 \mathcal{Z}_{k}^{+} \tilde{\Psi} \mathcal{Z}_{k}^{-}
\end{aligned}
$$

where $\tilde{\zeta}_{k}=\operatorname{vec}\left(\mathcal{Z}_{k}, \mathcal{Z}_{k}^{+}, \mathcal{Z}_{k}^{-}, \mathcal{W}_{k}\right)$ and $\tilde{\Pi}$ is a symmetrix matrix with entries given by

$$
\begin{aligned}
& \tilde{\Pi}_{1,1}=\mathcal{K}^{\top} P^{-1} \mathcal{K}-P^{-1}+\tilde{Q}_{1}, \tilde{\Pi}_{1,2}=\mathcal{K}^{\top} P^{-1} \mathcal{K}_{+}+\tilde{\Omega}_{+} \\
& \tilde{\Pi}_{1,3}=\mathcal{K}^{\top} P^{-1} \mathcal{K}_{-}+\tilde{\Omega}_{-}, \quad \tilde{\Pi}_{1,4}=\mathcal{K}^{\top} P^{-1} \tilde{D} \\
& \tilde{\Pi}_{2,2}=\mathcal{K}_{+}^{\top} P^{-1} \mathcal{K}_{+}+\tilde{Q}_{2}, \quad \tilde{\Pi}_{2,3}=\mathcal{K}_{+}^{\top} P^{-1} \mathcal{K}_{-}+\tilde{\Psi} \\
& \tilde{\Pi}_{2,4}=\mathcal{K}_{+}^{\top} P^{-1} \tilde{D}, \quad \tilde{\Pi}_{3,3}=\mathcal{K}_{-}^{\top} P^{-1} \mathcal{K}_{-}+\tilde{Q}_{3} \\
& \tilde{\Pi}_{3,4}=\mathcal{K}_{-}^{\top} P^{-1} \tilde{D}, \quad \tilde{\Pi}_{4,4}=\tilde{D}^{\top} P^{-1} \tilde{D}-\tilde{\Gamma}
\end{aligned}
$$

for any $\tilde{\Psi} \in \mathbb{R}^{2 n \times 2 n}$, non-negative definite $\tilde{Q}_{i}, \tilde{\Omega}_{+}, \tilde{\Omega}_{-} \in \mathbb{R}^{2 n \times 2 n}$, $i=\overline{1,3}$, and positive definite $\tilde{\Gamma}$. Then, if $\tilde{\Pi} \preceq 0$, we have that

$$
V_{k+1}-V_{k}=-\alpha V_{k}+\mathcal{W}_{k}^{\top} \tilde{\Gamma} \mathcal{W}_{k}
$$

meaning that $V_{k}$ is an ISS Lyapunov function, provided that $\tilde{Q}=$ $\tilde{Q}_{1}+\min \left\{\tilde{Q}_{2}, \tilde{Q}_{3}\right\}+2 \min \left\{\tilde{\Omega}_{+}, \tilde{\Omega}_{-}\right\} \succeq \alpha P^{-1}$ (such an $\alpha$ always exists if $\tilde{Q} \succ 0)$.

Decomposing $\tilde{\Pi}$ and applying the Schur complement (similarly as done for matrix $\Sigma$ in the proof of Theorem 1), the LMIs presented in this theorem are obtained by multiplying the resulting inequality by $\operatorname{diag}\{P, P, P, P, P\}$ from the left and right, and by introducing slack variables $\Gamma=P \tilde{\Gamma} P, \Omega_{+}=P \tilde{\Omega}_{+} P, \Omega_{-}=P \tilde{\Omega}_{-} P, \Psi=P \tilde{\Psi} P$, $\tilde{Q}_{i}=P Q_{i} P$, for $i=\overline{1,3}$, and by introducing new decision variables $W_{1}=K P, W_{2}=K_{+} P, W_{3}=K_{-} P$ and $W_{4}=R P$.

\section{PROOF OF COROLLARY 2}

First of all, the imposed inequalities on $Z$ and $\Gamma$ imply:

$$
\begin{gathered}
Z \geq \kappa P^{-1}, \frac{\eta}{\alpha} \Gamma \leq Z, \\
\frac{\eta}{\alpha} \Gamma \leq \kappa P \leq P Z P \Rightarrow \frac{\eta}{\alpha} \tilde{\Gamma}=\frac{\eta}{\alpha} P^{-1} \Gamma P^{-1} \leq Z,
\end{gathered}
$$

consequently, $\eta \mathcal{Z}_{k}^{\top} P^{-1} \mathcal{Z}_{k} \leq \frac{\eta}{\alpha} \mathcal{W}_{k}^{\top} \tilde{\Gamma} \mathcal{W}_{k} \leq \mathcal{W}_{k}^{\top} Z \mathcal{W}_{k} \leq 1$ for $\mathcal{Z}_{k} \in \tilde{\mathbb{X}}$. Next, note that $\mathcal{Z}_{k}=\mathcal{Z}_{k}^{+}-\mathcal{Z}_{k}^{-}$, then the control (16) can be rewritten as follows:

$$
u_{k}=\left(K+K_{+}\right) \mathcal{Z}_{k}^{+}+\left(K_{-}-K\right) \mathcal{Z}_{k}^{-}+R \mathcal{W}_{k},
$$

and the condition $u_{k} \in \mathbb{U}$ takes the form:

$$
\left[\begin{array}{c}
\mathcal{Z}_{k}^{+} \\
\mathcal{Z}_{k}^{-} \\
\mathcal{W}_{k}
\end{array}\right]^{\top}\left[\begin{array}{c}
K+K_{+} \\
K_{-}-K \\
R
\end{array}\right] S\left[\begin{array}{c}
K+K_{+} \\
K_{-}-K \\
R
\end{array}\right]^{\top}\left[\begin{array}{c}
\mathcal{Z}_{k}^{+} \\
\mathcal{Z}_{k}^{-} \\
\mathcal{W}_{k}
\end{array}\right] \leq 1 .
$$

If $\mathcal{Z}_{k} \in \tilde{\mathbb{X}}$, then obviously $\mathcal{Z}_{k}^{+}, \mathcal{Z}_{k}^{-} \in \tilde{\mathbb{X}}$, hence, the previous inequality is satisfied if

$$
\left[\begin{array}{c}
K+K_{+} \\
K_{-}-K \\
R
\end{array}\right] S\left[\begin{array}{c}
K+K_{+} \\
K_{-}-K \\
R
\end{array}\right]^{\top} \leq \frac{1}{3}\left[\begin{array}{ccc}
\eta P^{-1} & 0 & 0 \\
0 & \eta P^{-1} & 0 \\
0 & 0 & Z
\end{array}\right] .
$$

Then, (16) is obtained by applying the Schur complement in the relation above and then multiplying the resulting inequality from both sides by $\operatorname{diag}\left\{P, P, P, I_{m}\right\}$ and taking into account that $P Z P \geq \kappa P$.

\section{PROOF OF THEOREM 5}

First, note that the proposed MPC algorithm is based on conventional stabilizing ingredients, therefore this proof benefits of classic results [3]. Suppose that for any $\left[\underline{x}_{0}, \bar{x}_{0}\right] \subset \mathbb{X}$ a solution of OCP (17) exists, i.e., there is a sequence of inputs $\mathcal{S}_{N}^{0}$ that leads the trajectories of (10) to $\mathbb{X}_{f} \times \mathbb{X}_{f}$. This means that for $k=0$, by applying $u_{k}=s_{0}^{k}$, we ensure that $\left[\underline{z}_{k+1, i}, \bar{z}_{k+1, i}\right] \subset\left[\underline{z}_{k, i+1}, \bar{z}_{k, i+1}\right] \subset \mathbb{X}$ at least for $i=0$ and also $\left[\underline{z}_{k, N}, \bar{z}_{k, N}\right] \subset \mathbb{X}_{f}$. 
Then, following Algorithm 1, the procedure can be iteratively repeated for $k \in \mathbb{Z}_{+}$since $\left[\underline{w}_{k+1}, \bar{w}_{k+1}\right] \subseteq\left[\underline{w}_{k}, \bar{w}_{k}\right]$. Moreover, the control sequence $\mathcal{S}_{N}$ that steers $\left[\underline{z}_{k, 0}, \bar{z}_{k, 0}\right]$ to $\mathbb{X}_{f}$ also steers $x_{k}$ (as a consequence of (2)). This implies point (1).

For point (2), since the dynamics of $\mathcal{Z}_{k}$ is nominal (i.e., completely known) under Assumption 3, we have that ISS in $\mathbb{X}_{f}$ follows directly from the selection of the terminal ingredients (i.e., the choice of $\Psi_{j}$, $j=\{1,2,3\}$, in Algorithm 1), which guarantees that

$$
V\left(\mathcal{Z}_{k+1, N}\right)-V\left(\mathcal{Z}_{k, N}\right) \leq \mathcal{W}_{k}^{\top} \tilde{\Gamma} \mathcal{W}_{k}-\ell\left(\mathcal{Z}_{k, N}, s_{N}\right)
$$

due to (25) (see Section 3.3 in [3]). The practical ISS property for (1) follows the observations that

$$
\left|x_{k}\right| \leq\left|\mathcal{Z}_{k}\right|,\left|\mathcal{Z}_{0}\right| \leq\left|\underline{x}_{0}\right|+\left|\bar{x}_{0}\right| \leq|x(0)|+c
$$

where $c=\left|\underline{x}_{0}\right|+\left|\bar{x}_{0}\right|$ and $\mathcal{Z}_{k}=\operatorname{vec}\left(\underline{z}_{k, 0}, \bar{z}_{k, 0}\right)$.

Finally, for point (3), we have that the solution of OCP (17) implies that $x_{k} \in\left[\underline{z}_{k, 0}, \bar{z}_{k, 0}\right] \subset \mathbb{X}$ due to relation (2) and $u_{k}=s_{0}^{k} \in \mathbb{U}$, under Assumption 6 and the discussed features of $\mathbb{X}_{f}$.

\section{REFERENCES}

[1] A. Astolfi, D. Karagiannis, and R. Ortega, Nonlinear and Adaptive Control with Applications, ser. Communications on Control Engineering. Springer, 2008.

[2] R. Tóth, Modeling and Identification of Linear Parameter-Varying Systems, ser. Lecture Notes in Control and Information Sciences. Springer, 2010.

[3] D. Q. Mayne, J. B. Rawlings, C. V. Rao, and P. O. M. Scokaert, "Constrained model predictive control: stability and optimality," Automatica, vol. 36 , pp. $789-814,2000$.

[4] L. Grüne and J. Pannek, Nonlinear Model Predictive Control: Theory and Algorithms, ser. Communications and Control Engineering. Springer-Verlag London, 2011.

[5] A. Bemporad and M. Morari, "Robust model predictive control: A survey," in Robustness in Identification and Control, 1998

[6] B. Kouvaritakis and M. Cannon, Eds., Model Predictive Control: Classical, Robust and Stochastic. Springer, 2016.

[7] M. M. Morato, J. Normey-Rico, and O. Sename, "Model predictive control design for linear parameter varying systems: A survey," Annual Reviews in Control, 2020.

[8] B. Ding, "Constrained robust model predictive control via parameterdependent dynamic output feedback," Automatica, vol. 46, no. 9, pp. $1517-1523,2010$.

[9] B. Ding, X. Ping, and H. Pan, "On dynamic output feedback robust MPC for constrained quasi-LPV systems," International Journal of Control, vol. 86, no. 12, pp. 2215-2227, 2013.

[10] W. Yang, J. Gao, G. Feng, and T. Zhang, "An optimal approach to output-feedback robust model predictive control of LPV systems with disturbances," International Journal of Robust and Nonlinear Control, vol. 26 , no. 15 , pp. 3253-3273, 2016.

[11] X. Ping, S. Yang, P. Wang, and Z. Li, "An observer-based output feedback robust MPC approach for constrained LPV systems with bounded disturbance and noise," International Journal of Robust and Nonlinear Control, vol. 30, no. 4, pp. 1512-1533, 2020.

[12] Y. Yang, B. Ding, Z. Xu, and J. Zhao, "Tube-based output feedback model predictive control of polytopic uncertain system with bounded disturbances," Journal of the Franklin Institute, vol. 356, no. 15, pp. 7990-8011, 2019.

[13] H. Huang, D.-F. He, and Q.-X. Chen, "Quasi-min-max dynamic output feedback MPC for LPV systems," Int. J. System Control and Information Processing, vol. 1, no. 3, 2014.

[14] T. Kim, J. Park, and T. Sugie, "Output-feedback model predictive control for LPV systems with input saturation based on quasi-min-max algorithm," in Proceedings of the 45th IEEE Conference on Decision and Control, 2006, pp. 1454-1459.

[15] B. Ding, P. Wang, and J. Hu, "Dynamic output feedback robust MPC with one free control move for LPV model with bounded disturbance," Asian Journal of Control, vol. 20, no. 2, pp. 755-767, 2018.

[16] B. Ding and H. Pan, "Output feedback robust MPC for LPV system with polytopic model parametric uncertainty and bounded disturbance," International Journal of Control, vol. 89, no. 8, pp. 1554-1571, 2016.
[17] A. Reis de Souza, D. Efimov, T. Raïssi, and X. Ping, "Robust output feedback MPC: An interval-observer approach," in Proc. IEEE Conference on Decision and Control (CDC), Jejju, South Korea, 2020.

[18] J. Gouzé, A. Rapaport, and M. Hadj-Sadok, "Interval observers for uncertain biological systems," Ecological Modelling, vol. 133, no. 1, pp. $45-56,2000$.

[19] T. Raïssi and D. Efimov, "Some recent results on the design and implementation of interval observers for uncertain systems," Automatisierungstechnik, vol. 66, no. 3, pp. 213 - 224, 2018.

[20] D. Efimov, W. Perruquetti, T. Raïssi, and A. Zolghadri, "On interval observer design for time-invariant discrete-time systems," in $2013 \mathrm{Eu}$ ropean Control Conference (ECC), 2013, pp. 2651-2656.

[21] D. Efimov and T. Raïssi, "Design of interval observers for uncertain dynamical systems," Autom. Remote Control, vol. 77, no. 2, pp. 191225, Feb. 2016

[22] L. Farina and S. Rinaldi, Positive Linear Systems: Theory and Applications. John Wiley \& Sons, 2000.

[23] Y. Wang, Z. Wang, V. Puig, and G. Cembrano, "Zonotopic setmembership state estimation for discrete-time descriptor lpv systems," IEEE Transactions on Automatic Control, vol. 64, no. 5, pp. 2092-2099, 2019.

[24] S. Boyd and L. Vanderberghe, Convex Optimization. Cambridge University Press, 2004.

[25] S. Di Cairano, H. Tseng, D. Bernardini, and A. Bemporad, "Steering vehicle control by switched model predictive control," IFAC Proceedings Volumes, vol. 43, no. 7, pp. $1-6,2010$.

[26] J. Löfberg, "Yalmip : A toolbox for modeling and optimization in matlab," in In Proceedings of the CACSD Conference, Taipei, Taiwan, 2004.

[27] M. Herceg, M. Kvasnica, C. Jones, and M. Morari, "Multi-Parametric Toolbox 3.0," in Proc. of the European Control Conference, Zürich, Switzerland, July 17-19 2013, pp. 502-510, http://control.ee.ethz.ch/ $\sim$ mpt. 